\title{
Experimental Study of Convex Coded Synthetic Transmit Aperture Imaging
}

\author{
Gammelmark, Kim; Jensen, Jørgen Arendt
}

Published in:

IEEE Ultrasonics Symposium, 2002. Proceedings

Link to article, DOI:

10.1109/ULTSYM.2002.1192603

Publication date:

2002

Document Version

Publisher's PDF, also known as Version of record

Link back to DTU Orbit

Citation (APA):

Gammelmark, K., \& Jensen, J. A. (2002). Experimental Study of Convex Coded Synthetic Transmit Aperture Imaging. In IEEE Ultrasonics Symposium, 2002. Proceedings (pp. 1573-1576). IEEE.

https://doi.org/10.1109/ULTSYM.2002.1192603

\section{General rights}

Copyright and moral rights for the publications made accessible in the public portal are retained by the authors and/or other copyright owners and it is a condition of accessing publications that users recognise and abide by the legal requirements associated with these rights.

- Users may download and print one copy of any publication from the public portal for the purpose of private study or research

- You may not further distribute the material or use it for any profit-making activity or commercial gain

- You may freely distribute the URL identifying the publication in the public portal

If you believe that this document breaches copyright please contact us providing details, and we will remove access to the work immediately and investigate your claim. 


\title{
Experimental Study of Convex Coded Synthetic Transmit Aperture Imaging
}

\author{
Kim Løkke Gammelmark and Jørgen Arendt Jensen* \\ Center for Fast Ultrasound Imaging, Ørsted•DTU, Build. 348, \\ Technical University of Denmark, 2800 Kgs. Lyngby, Denmark
}

\begin{abstract}
Synthetic transmit aperture imaging is investigated using a convex array transducer. To increase the signalto-noise ratio, a multi-element subaperture is used to emulate the spherical wave transmission, and the conventional short excitation pulse is replaced by a linear FM signal. The approach is compared to the conventional application of the convex array in commercial scanners. The array used is a commercial $5.5 \mathrm{MHz}, 128$ element array with $60 \%$ bandwidth and $\lambda$ pitch. For conventional imaging a 64 element transmit aperture is used with a 2 cycle temporally weighted sinusoid as excitation signal. For synthetic aperture imaging an 11 element transmit aperture is used with a $20 \mu$ s linear FM signal as excitation. For both methods, 128 elements are used on receive. Measurements are done using our experimental multi-channel ultrasound scanner, RASMUS. Wire phantom measurements show an improvement in lateral resolution of about $30 \%$ throughout the image with lower near and far field sidelobe levels. Results from a cyst phantom show big improvements in contrast resolution, and an increase in penetration depth of about $2 \mathrm{~cm}$. In-vivo images of the abdomen of a healthy 27 year old male show slight improvements in image quality, especially in the near field.
\end{abstract}

\section{INTRODUCTION}

It is widely accepted that synthetic transmit aperture (STA) imaging can be used to obtain images with better image quality compared to conventional techniques like phased and linear array imaging. The reason for this is that both transmit and receive beamforming can be applied in STA imaging, while only a single transmit focus combined with receive beamforming is used in the traditional imaging techniques. Consequently, STA beamforming is done using dynamic focusing and apodization in both transmit and receive, which gives better control of the beam shape. However, it is also well known that STA imaging suffers from a low signal-tonoise ratio (SNR) due to the single element transmissions.

*This work was supported by grants 9700883 and 9700563 from the Danish Science Foundation, by Ph.D. grant 11-3160-55173 from the Technical University of Denmark, and by B-K Medical A/S, Herlev, Denmark.
Recent research has though shown [1] that the combination of existing techniques of multi-element subapertures and temporal encoding can be utilized to increase the SNR to exceed that of the conventional imaging techniques.

Traditionally, STA imaging has been considered for phased array applications [2] and recently linear array imaging [1]. However, only little research on the application of STA imaging with convex array transducers has been published in the open literature [3]. Convex array transducers are generally used for abdominal imaging because of their large field of view and good penetration depth. Extending STA imaging to convex apertures requires some new design considerations because of the more complicated aperture geometry. The advantage is, however, better abdominal images than those obtained by linear and phased arrays, because the convex array is designed specifically for this application.

This paper investigates STA imaging using multi-element subapertures and temporal encoding for a convex transducer array. The method is described in Section II, and the set-up for the measurement using our experimental multi-channel ultrasound scanner, RASMUS, are described in Section III. The measurement results for both phantom objects and invivo imaging are presented in Section IV.

\section{THEORY}

In conventional STA imaging a single element is used at each transmission to produce a spherical wave that interrogates a large region of the medium. The echoes are recorded using all elements in the aperture, and the procedure is repeated until a desired number of elements have been used for transmission. For each transmission, the received echoes contain information about all scatterers in the interrogated region. Since no focusing has been applied on transmit, and the exact origin of the spherical wave is known, the receive aperture can be steered in any direction and focused at any point within this region. Therefore, every point in the image can be formed independent of each other, and a full image is obtained for every transmission event with low lateral resolution due to the single transmit element. Taking into account 
Transmit aperture
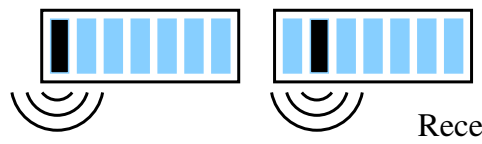

Receive aperture

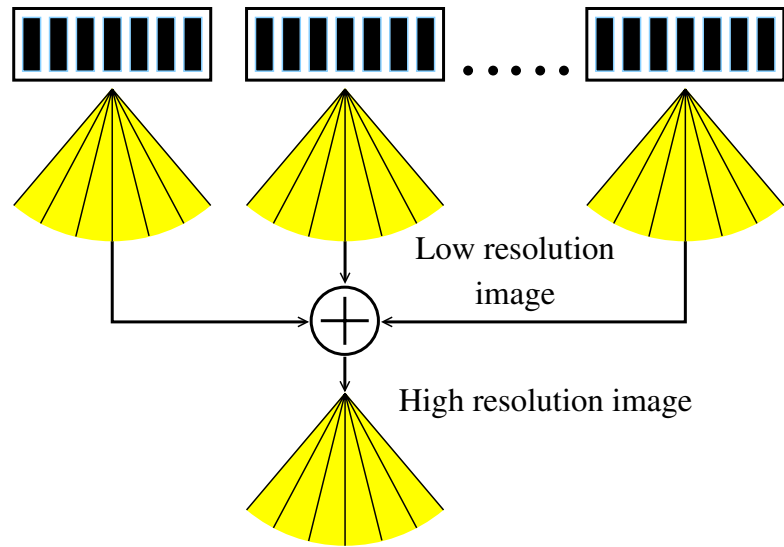

Figure 1: Illustration of the conventional STA imaging principle. At each transmission event a single element is fired, and the echoes from the medium are collected using all elements in the receiving aperture. For each of these transmission events a complete image is beamformed, and these are subsequently summed to form the final high resolution image.

the full path length between the transmitting element and the receive elements, when calculating the beamforming delays, the individual low resolution images are both dynamically focused on receive, and adjusted to obtain dynamic focusing on transmit in the same operation. Dynamic focusing on transmit is then obtained subsequently by coherently summing the low resolution images which forms the final high resolution image as illustrated in Fig. 1.

The STA imaging approach investigated in this paper combines multi-element STA imaging and linear FM signals with a convex array transducer. This approach has recently been investigated using linear array transducers [1], where the results showed significant improvements in both spatial and contrast resolution. In general, the concept of STA imaging does not depend on the array type, however, the more complicated geometry of the convex array makes it necessary to redesign the use of multi-element subapertures. This is described below.

The multi-element subaperture is utilized to produce a high power spherical wave over a limited angular extend. Traditionally, this is done by placing a virtual point source at a distance $r$ behind the center of the subaperture as illustrated in the top part of Fig. 2. The spherical wave produced is symmetric with an angular extend $\alpha$, which is determined by $r$.

Due to the geometry of the array and the shape of the

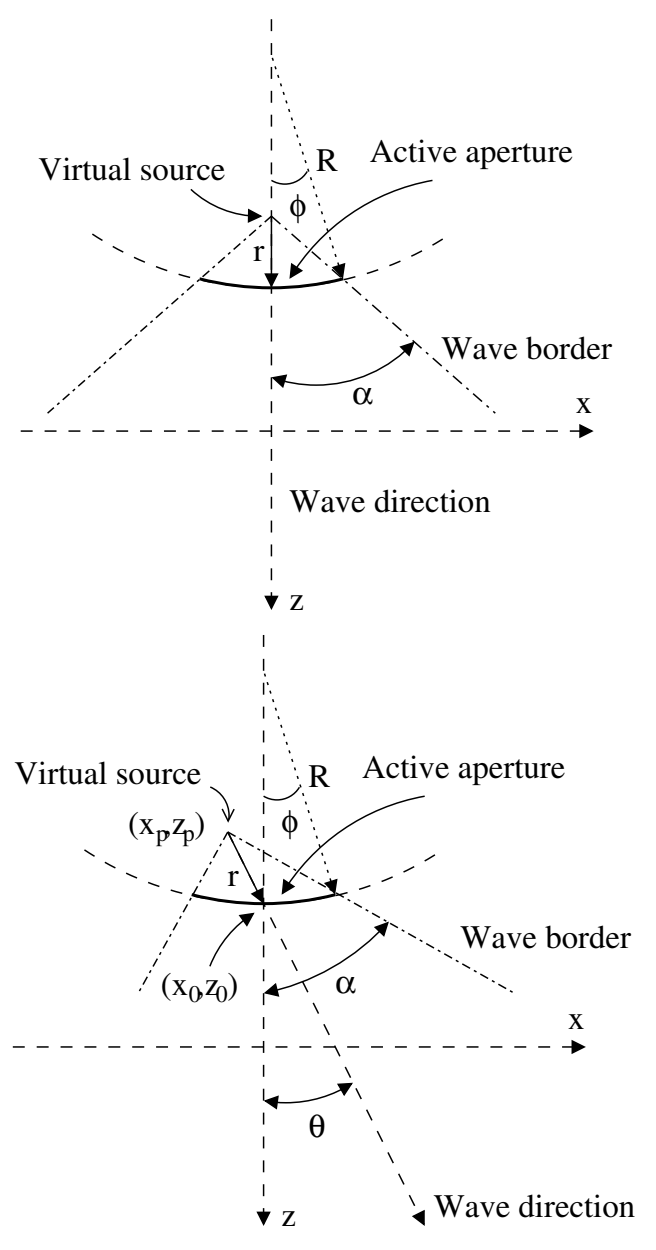

Figure 2: Illustration of two methods for applying multielement STA imaging to convex arrays. Top: Conventional application where the virtual point source is placed behind the center of the subaperture to produce a symmetric spherical wave. Bottom: Angled transmission where the virtual point source is placed such that the spherical wave propagates at an angle $\theta$.

convex array images, a significant part of the energy in the spherical wave will propagate outside the image area when the outer subapertures are used. Thus, to limit the amount of wasted energy, the transmissions can be angled as illustrated in the bottom part of Fig. 2. Here, the virtual point source is moved to the side to obtain a wave direction $\theta$, and at the same time increase $\alpha$. It is noted, that the wave is no longer symmetric and that the angular extend of the wave $\alpha$ is larger in the direction of the beam. The position of the virtual point source is calculated geometrically as

$$
\left[\begin{array}{c}
z_{p} \\
x_{p}
\end{array}\right]=\left[\begin{array}{c}
z_{0}-r \cdot \cos \theta \\
x_{0}-r \cdot \sin \theta
\end{array}\right],
$$


where $r$ is the radial distance to the virtual source given by

$$
r=R\left(\frac{\sin \phi-\tan \alpha(\cos \phi-1)}{\tan \alpha \cos |\theta|-\sin |\theta|}\right),
$$

and $\phi$ is half the angular size of the subaperture. Note, that $\alpha$ and $\phi$ are always positive, and that the coordinate system used here moves with the subaperture. Also, the directivity pattern of the individual elements will have an influence on the shape of the wave, but this has not been included in the model at this point.

Using this approach the individual transmissions can be directed such that the spherical wave interrogates a larger region of the image, and thus more image points can be beamformed for each transmission. In this study, the wave direction for each transmission is chosen such that the center of the spherical wave travels parallel to the axis of the transducer, i.e. straight down.

\section{Measurement Setup}

The measurements are performed using the experimental multi-channel ultrasound scanning system, RASMUS [4]. The system has 128 individually programmable transmitters capable of sending arbitrary coded waveforms with a precision of 12 bits at $40 \mathrm{MHz}$. Sixty-four receive channels can be sampled simultaneously at 12 bits and $40 \mathrm{MHz}$, and the 2-to-1 multiplexing in the system enables acquisition of 128 channels in real-time over two transmissions. The system has 16 Gbytes of storage memory in the receivers, which enables the acquisition of several seconds of RF data. The RASMUS system is remotely accessible and programmed through a developed Matlab/C-library interface.

The transducer used for the measurements is a commercial 5.5 MHz convex array transducer with 192 elements and $\lambda$ pitch. The bandwidth of the elements is $60 \%$ relative to the center frequency, and the convex radius of the aperture is 41 $\mathrm{mm}$. The height of the elements is $8 \mathrm{~mm}$, and the array has a lens in the elevation dimension with a fixed focus at $40 \mathrm{~mm}$. Due to the limitation of the RASMUS system, only the central 128 elements of the array are used.

For conventional imaging a 64 elements, Hamming weighted, transmit aperture is used with a fixed focus at 70 mm. A 2 cycle sinusoid at $5.5 \mathrm{MHz}$ weighted with a Hanning window is used as the excitation pulse, and all 128 elements are used on receive and for the subsequent beamformation. For synthetic imaging an 11 element subaperture is used to emulate the spherical wave transmission, and all 128 elements in the aperture are used on receive. The center of the transmit aperture is moved one element after each transmission, which results in a total of 118 emissions. A $20 \mu$ s linear FM signal with a center frequency of $5.5 \mathrm{MHz}$ and $90 \%$ bandwidth is used as excitation signal. The FM signal has been weighted with a Tukey window to reduce the temporal sidelobes [5].

The conventional data are beamformed using dynamic receive focusing with delay updates for each sample. The RF signals are filtered using a matched filter, and dynamic Hamming apodization is applied which opens the receive aperture at a f-number of 2. The edge level of the Hamming window is raised to $-12 \mathrm{~dB}$. The synthetic data are processed using dynamic transmit and receive focusing. A designed compression filter with a center frequency of $5.5 \mathrm{MHz}$ and $90 \%$ bandwidth is used to filter the RF data. The filter is weighted with a Chebychev window to reduce the near and distant temporal sidelobes in the compressed signal $[5,1]$. Dynamic Hamming apodization is applied in both transmit and receive with -12 $\mathrm{dB}$ edge levels, and both apertures are opened at a f-number equal to 2 . When beamforming both sets of data the element sensitivity is taken into account. The acceptance angle [6] is set to \pm 50 degrees for each element, and, thus, an element is not applied in the beamformer until the point to be beamformed lies inside its acceptance angle.

\section{Results \& CONCLUSion}

A wire phantom with an attenuation of $0.5 \mathrm{~dB} /[\mathrm{MHz} \cdot \mathrm{cm}]$ was scanned to investigate the spatial resolution and penetration depth of the approach. The results are shown in Fig. 3 for conventional scanning to the left and synthetic imaging to the right. The dynamic range in both images is $45 \mathrm{~dB}$. The improvement in lateral resolution for synthetic imaging is noticed throughout the image, especially at the group of wires at $90 \mathrm{~mm}$. The row of wires at $140 \mathrm{~mm}$ is barely visible in the synthetic image but not in the conventional image, which indicates a slight improvement in penetration depth. The axial resolution has, however, been slightly degraded in the synthetic image. Thus, the design of the linear FM signal and the corresponding compression filter needs to be revised, since the bandwidth and weighting functions highly affect the compression efficiency [7].

To investigate the contrast resolution a cyst phantom was scanned. The phantom contains tissue mimicking material with an attenuation of $0.5 \mathrm{~dB} /[\mathrm{MHz} \cdot \mathrm{cm}]$ and 5 columns of anechoic cysts. The cysts in the different columns have diameters of $16,8,4,2,1 \mathrm{~mm}$ from left to right, and the columns are placed at an angle of 15 degrees. The attenuation in the phantom is $0.5 \mathrm{~dB} /[\mathrm{MHz} \cdot \mathrm{cm}]$. The results are shown in Fig. 4 for conventional imaging (left) and synthetic imaging (right). The improvement in both contrast resolution and penetration depth is evident and significant improvements are obtained below $90 \mathrm{~mm}$.

In-vivo images of the abdomen of a healthy 27 year old male volunteer were obtained to investigate clinical improvements in spatial and contrast resolution. The results are shown in Fig. 5 with the conventional method displayed to the left and the synthetic approach to the right. The dynamic 

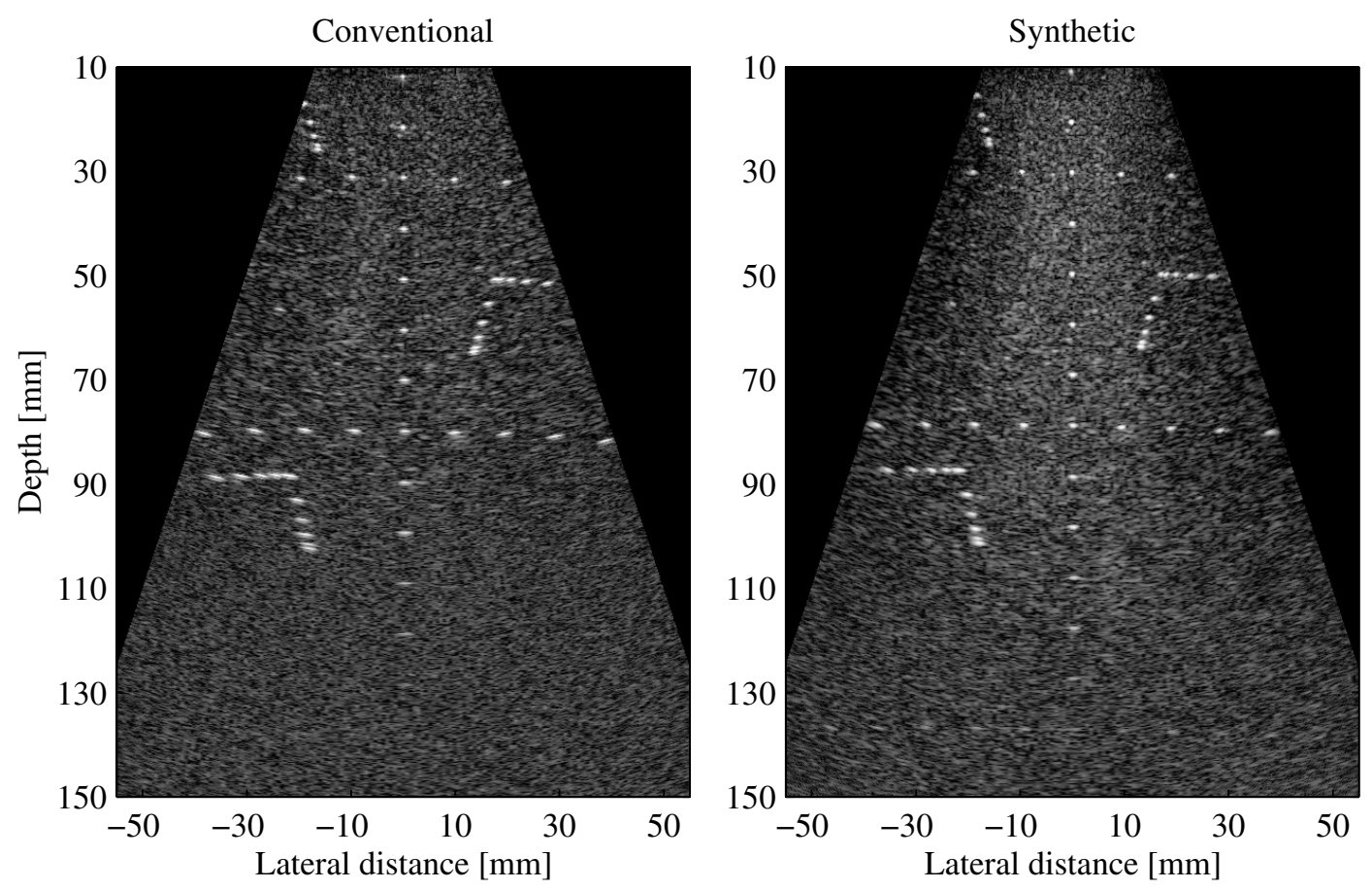

Figure 3: Measured images of a wire phantom with an attenuation of $0.5 \mathrm{~dB} /[\mathrm{MHz} \cdot \mathrm{cm}]$. The conventional image is displayed to the left and the synthetic image to the right. The dynamic range in both images is $45 \mathrm{~dB}$.

range in both images is $40 \mathrm{~dB}$. The portal vein branch is seen in the center of the image with the hepatic vein located above to the left and the caval vein below to the right with respect to the portal vein. A slightly better spatial and contrast resolution seems to have been obtained. This is especially noticed at the small vessels in the center of the image. There are, however, also small distortions in the synthetic image as seen at the caval vein in the left part of the image. This may be due to the influence of tissue motion and phase aberration to which STA imaging is more susceptible than conventional imaging. These issues have not been addressed in this study and, thus, they need to be considered in the future work to obtain the image quality improvements shown in the phantom images.

\section{REFERENCES}

[1] K. L. Gammelmark and J. A. Jensen, "Multi-element synthetic transmit aperture imaging using temporal encoding," in Proc. SPIE: Progress in biomedical optics and imaging, 2002, vol. 3, pp. 25-36.

[2] M. Karaman, P. C. Li, and M. O'Donnell, "Synthetic aperture imaging for small scale systems," IEEE Trans. Ultrason., Ferroelec., Freq. Contr., vol. 42, pp. 429-442, 1995.

[3] J. T. Ylitalo, "Synthetic aperture ultrasound imaging us- ing a convex array," in Proc. IEEE Ultrason. Symp., 1995, pp. 1337-1340.

[4] J. A. Jensen, O. Holm, L. J. Jensen, H. Bendsen, H. M. Pedersen, K. Salomonsen, J. Hansen, and S. Nikolov, "Experimental ultrasound system for real-time synthetic imaging," in Proc. IEEE Ultrason. Symp., 1999, vol. 2, pp. $1595-1599$.

[5] T. X. Misaridis and J. A. Jensen, "An effective coded excitation scheme based on a predistorted FM signal and an optimized digital filter," in Proc. IEEE Ultrason. Symp., 1999, vol. 2, pp. 1589-1593.

[6] M. O'Donnell and L. J. Thomas, "Efficient synthetic aperture imaging from a circular aperture with possible application to catheter-based imaging," IEEE Trans. Ultrason., Ferroelec., Freq. Contr., vol. 39, pp. 366-380, 1992.

[7] T. Misaridis, Ultrasound imaging using coded signals, Ph.D. thesis, Ørsted•DTU, Technical University of Denmark, Lyngby, Denmark, 2001. 

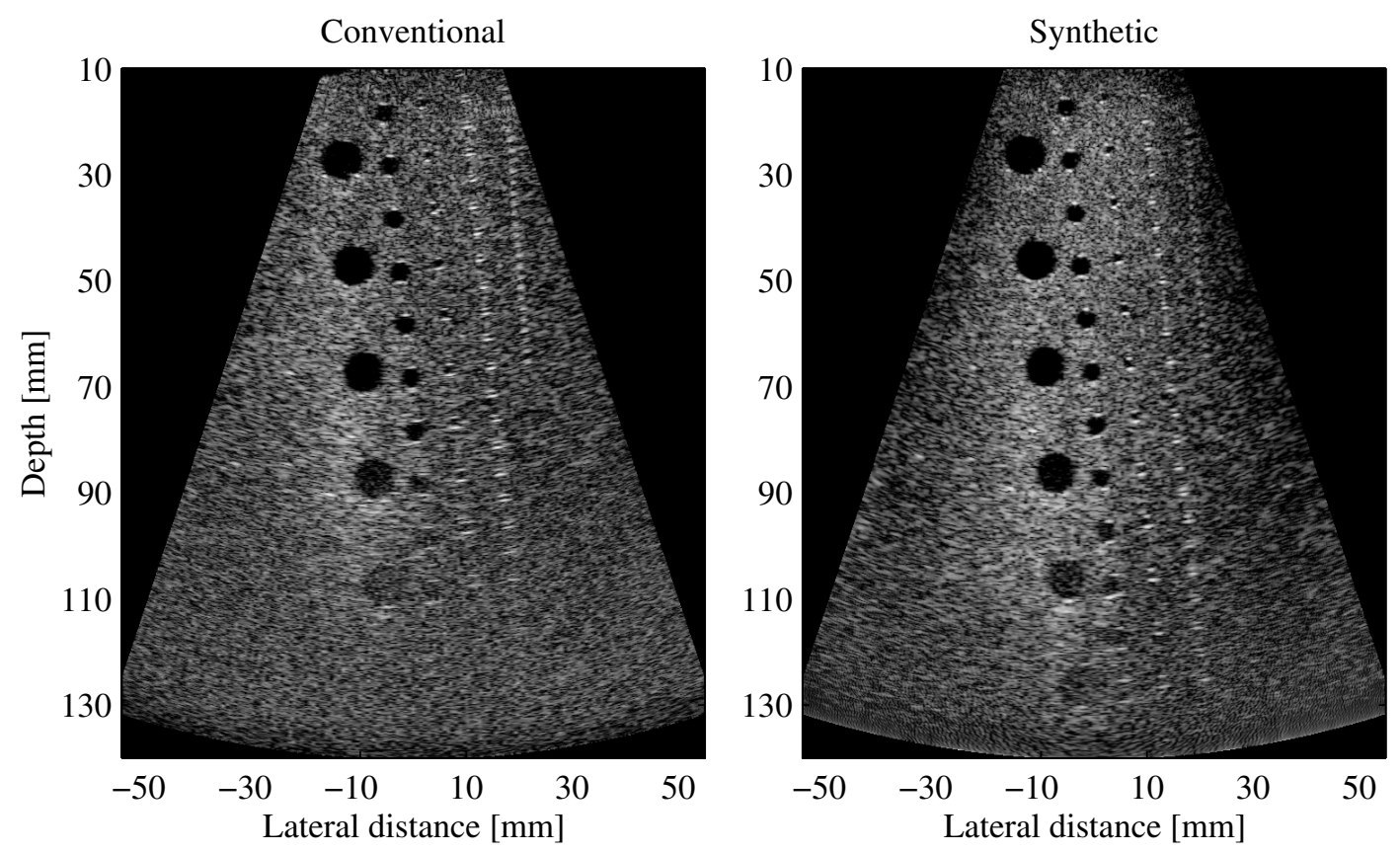

Figure 4: Measured images of a cyst phantom with an attenuation of $0.5 \mathrm{~dB} /[\mathrm{MHz} \cdot \mathrm{cm}]$. The conventional image is displayed to the left and the synthetic image to the right. The dynamic range in both images is $40 \mathrm{~dB}$.
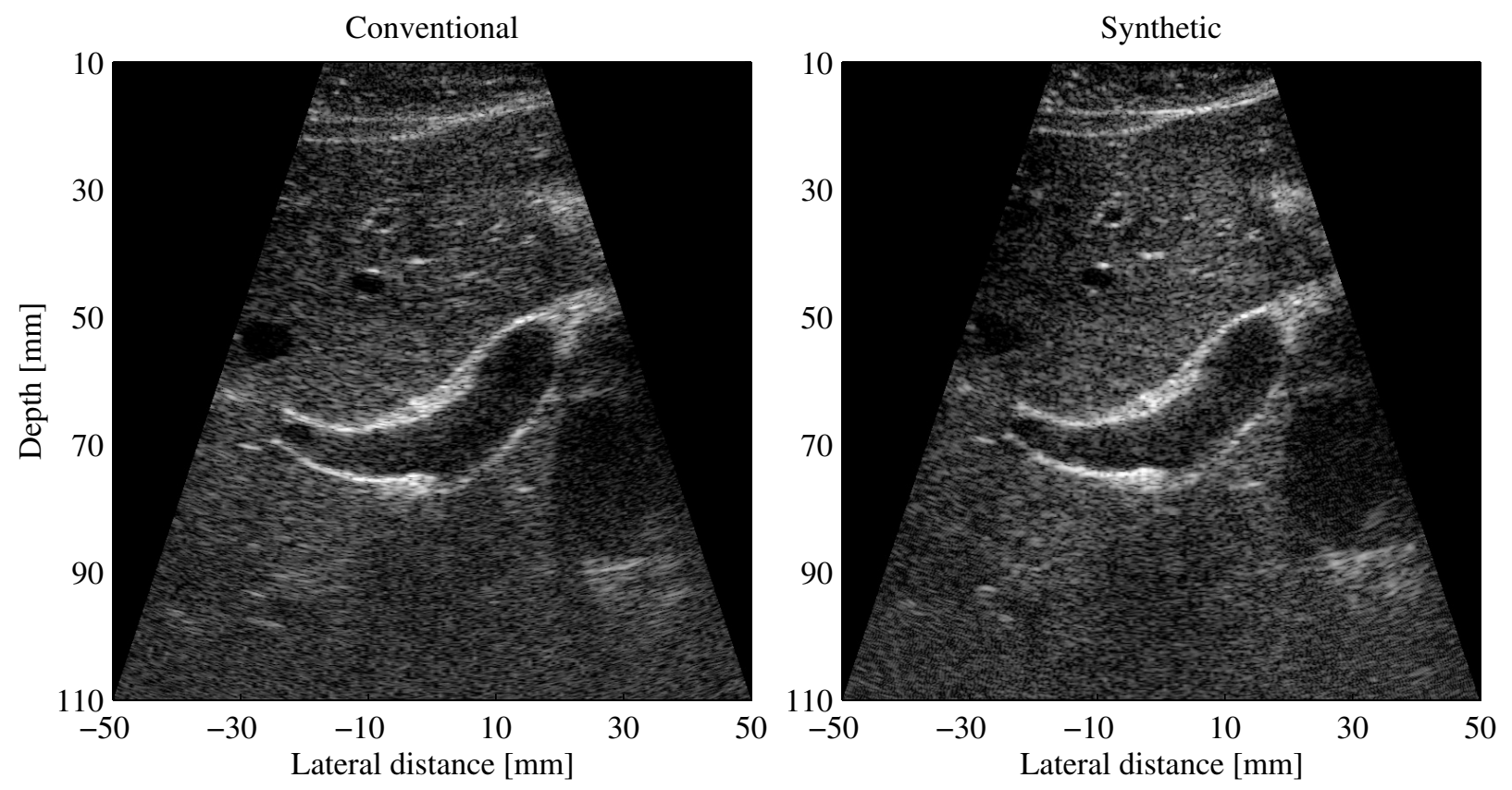

Figure 5: Measured images of the abdomen of a healthy 27 year old volunteer. The portal vein branch is seen in the center of the images, and the hepatic and caval veins are located to the left and right of the portal vein, respectively. The conventional image is shown to the left, and the synthetic image is shown to the right. The dynamic range is $40 \mathrm{~dB}$ in both images. 